\title{
An atomistic investigation into the nature of near threshold fatigue crack growth in aluminum alloys
}

\author{
K.L. Baker ${ }^{1}$, D.H. Warner* \\ School of Civil and Environmental Engineering, Cornell University, Ithaca, NY 14853, United States
}

\section{A R T I C L E I N F O}

\section{Article history:}

Received 26 February 2013

Received in revised form 21 October 2013

Accepted 28 October 2013

Available online 8 November 2013

\section{Keywords:}

Threshold fatigue

Aluminum alloy

Multiscale simulation

Crack growth mechanisms

\begin{abstract}
A B S T R A C T
Despite decades of study, the atomic-scale mechanisms of fatigue crack growth remain elusive. Here we use the coupled atomistic-discrete dislocation method, a multiscale simulation method, to examine the influence of dislocation glide resistance on near-threshold fatigue crack growth in an aluminum alloy. The simulations indicate that the threshold increases with an increase in dislocation glide resistance, and that a transition in the crack growth direction can occur when dislocation nucleation is inhibited by other nucleated dislocations. Three main mechanisms of fatigue crack propagation are observed: cleavage along the primary slip plane, crack extension by dislocation emission, and crack extension by opening along lattice defects.
\end{abstract}

(c) 2013 Elsevier Ltd. All rights reserved.

\section{Introduction}

Aluminum alloys continue to serve as the primary material system for many critical components in aircraft structures. Accordingly, a key aspect of aircraft safety involves the prediction of fatigue crack growth in these materials. This technological motivation has spurred the growth of a vast library of experimental and theoretical studies on fatigue crack growth over the past decades. Nonetheless, one critical aspect of the phenomenon has remained particularly unclear, i.e. the atomistic mechanism by which the crack tip propagates forward under cyclic subcritical loadings.

Considering the atomic nature of crack tip processes, modeling must be atomistic in nature. However, interpreting atomistic modeling results relative to fatigue crack growth in real alloys involves many significant challenges. One of the largest challenges is the limited spatial domain that is typical of atomistic models. Simulations having a small spatial domain can artificially influence the movement of dislocations away from the crack tip and ultimately bias crack tip behavior [1-8]. Discrete dislocation (DD) dynamics simulations are not generally plagued by this problem as they can accommodate a much larger spatial domain, while still explicitly modeling every dislocation. However, DD models do not explicitly represent the atomic scale complexities that occur at a crack tip [9-13], and thus cannot illuminate the atomic mechanisms by which a crack tip propagates.

In this work, a concurrently coupled atomistic-discrete dislocation multiscale method (CADD) is used to resolve the shortcomings of tradition atomistic and DD simulations with the specific goal of illuminating the atomic scale mechanisms that occur at a crack tip during fatigue crack growth. The model consists of an aluminum crystal with a crack, loaded in mode I. Upon loading, dislocations nucleate at the crack tip in the atomistic region of the model. In most cases, the dislocations then glide into the nearby DD region, where their motion is inhibited by an imposed glide resistance (friction force). The imposed glide resistance is the primary variable studied in this work, and is the sole feature of the model that connects it

\footnotetext{
* Corresponding author. Tel.: +1 (607) 2557155.

E-mail addresses: klb244@cornell.edu (K.L. Baker), dhw52@cornell.edu (D.H. Warner).

1 Present address: École Polytechnique Fédérale de Lausanne, CH-1015 Lausanne, Switzerland.
} 


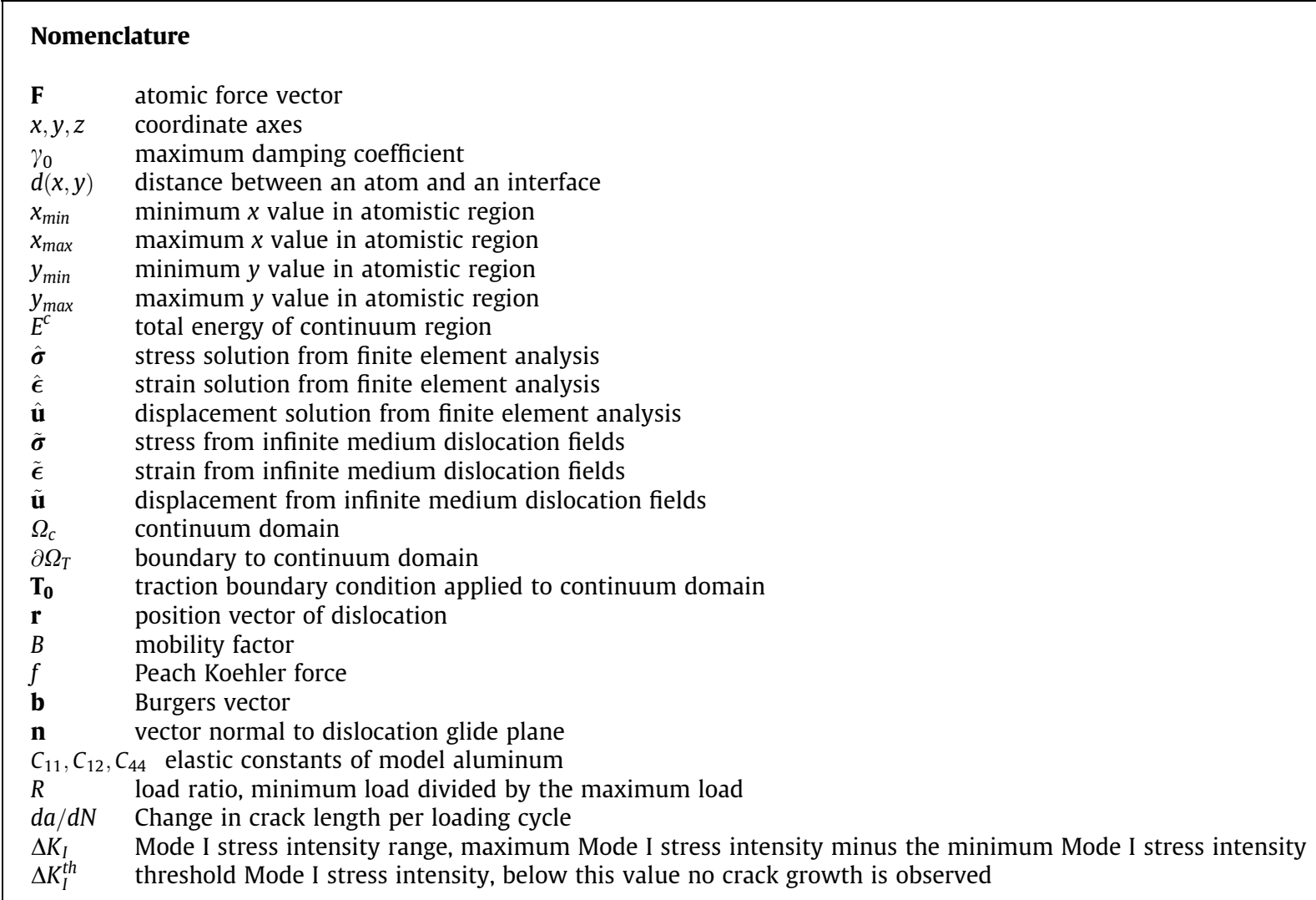

to aluminum alloy response, i.e. no alloying elements are included in the atomistic region and thus direct interactions between the crack tip and alloying elements and precipitates are not considered. By studying the relationship between dislocation glide resistance and crack tip response under cyclic loading, this work aims to illuminate the effect of aging on fatigue crack growth, something for which experimental results exist.

The manuscript focuses on two key components of the results emerging from our models. The first is the identification of the atomistic mechanisms by which a crack tip propagates forward in a ductile metal subjected to cyclic loading. The second is the dependence of the crack tip mechanisms on dislocation glide resistance, which gives insight into the mechanism by which experimentally observed near threshold fatigue crack growth rates are influenced by the aging of alloys.

\section{Methods}

A concurrent multiscale simulation approach is used to connect an atomistic region encompassing an aluminum crack tip with a discrete dislocation region capable of capturing dislocation pileups microns in length. In this way, the detailed atomistic processes occurring at the crack tip can be simulated under the presence of a realistic stress filed created by a distribution of dislocations. Dislocations on the primary slip plane are restricted from gliding freely away from the crack tip by a friction force applied homogeneously throughout the discrete dislocation portion of the simulation.

\subsection{CADD concurrent multiscale method}

The underlying theory of the multiscale method is built upon the coupled atomistic-discrete dislocation (CADD) framework of Shilkrot et al. [14,15]. The CADD coupling methodology consists of solving two distinct problems, involving an atomistic and continuum region that are coupled by self-consistent displacement boundary conditions. The atomistic region is composed of a set of atoms bounded by a set of interface atoms/nodes as seen in Fig. 1. The DD continuum region is approximated by finite elements and thus the fields associated with it are a function of the corresponding nodes. The interatomic force, $\mathbf{F}$, experienced by each atom within the atomistic region and at the interface results from interactions not only within the atomistic region and its bounding interface, but also interactions with pad atoms that extend into the continuum. The forces are then used to dynamically relax the atomic positions. Unlike the atoms in the atomistic and interface regions, 
(a)

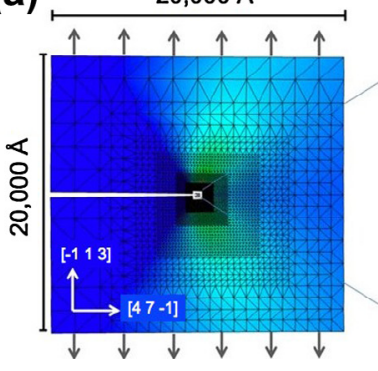

(b)

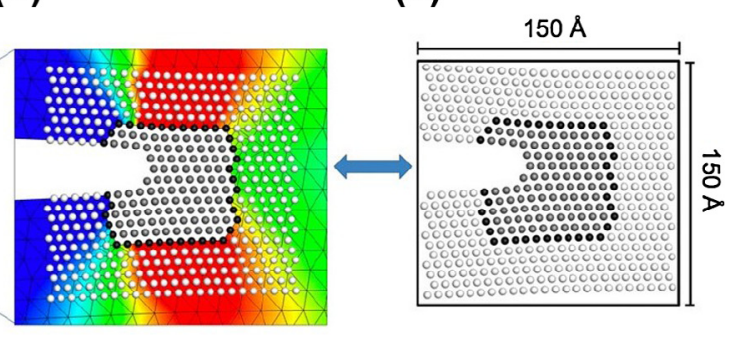

Fig. 1. A schematic diagram of a CADD model. (a) The continuum problem with applied far-field boundary conditions. In this case an edge crack. (b) The coupling of the continuum and atomistic regions at a boundary of coincident points, i.e. interface atoms (black). The pad atoms (white) exist in the continuum region and their displacement is controlled by the continuum displacement field. (c) The atomistic problem with the interface and pad atoms held fixed to apply the correct, non-local boundary conditions.

the positions of the pad atoms are controlled by the continuum solution. Thus the pad atoms provide the necessary non-local boundary conditions across the interface to the atomistic and DD regions. While the CADD methodology is not specific to any particular interatomic force model [16,17], this work utilizes embedded atom empirical potentials to model interatomic forces [18].

The response of the continuum region is local, and therefore, it is only a function of the boundary conditions and the resulting deformation within that region. The continuum region is modeled to be constantly in static equilibrium, such that the energy functional associated with the region is always minimized,

$$
E^{c}=\frac{1}{2} \int_{\Omega_{c}}(\hat{\boldsymbol{\sigma}}+\tilde{\boldsymbol{\sigma}}):(\hat{\boldsymbol{\epsilon}}+\tilde{\boldsymbol{\epsilon}}) \mathrm{d} V-\int_{\partial \Omega_{T}} \mathbf{T}_{\mathbf{0}}(\hat{\mathbf{u}}+\tilde{\mathbf{u}}) \mathrm{d} A,
$$

where $\Omega_{c}$ represents the continuum domain and $\partial \Omega_{T}$ represents the boundary of the continuum domain on which a traction boundary condition, $\mathbf{T}_{\mathbf{0}}$, exists. $\tilde{\boldsymbol{\sigma}}, \tilde{\boldsymbol{\epsilon}}$, and $\tilde{\mathbf{u}}$, represent the infinite medium stress, strain, and displacement fields, respectively, for the discrete dislocations at their distinct locations. $\hat{\boldsymbol{\sigma}}, \hat{\boldsymbol{\epsilon}}$, and $\hat{\mathbf{u}}$ represent the solution fields from an anisotropic linear elastic boundary value problem that when superimposed with the dislocation fields satisfy the overall boundary conditions. $\hat{\boldsymbol{\sigma}}, \hat{\boldsymbol{\epsilon}}$, and $\hat{\mathbf{u}}$ are obtained using a standard displacement based finite element approach, while $\tilde{\boldsymbol{\sigma}}, \tilde{\boldsymbol{\epsilon}}$, and $\tilde{\mathbf{u}}$ are well known analytic fields [19]. The position of the dislocations within continuum domain evolve following

$$
\mathbf{r}_{\text {new }}^{i}=\mathbf{r}_{\text {old }}^{i}+B\left(f^{i} \frac{\mathbf{b}^{i}}{\left|\mathbf{b}^{i}\right|}\right)
$$

where $\mathbf{r}_{\text {new }}^{i}$ is the new position of dislocation $i, \mathbf{r}_{\text {old }}^{i}$ is the position of dislocation $i$ from the previous update step, $\mathbf{b}^{i}$ is the Burgers vector of dislocation $i, B$ is the mobility factor, and $f^{i}$ is the Peach Koehler force of dislocation $i$ given by

$$
f^{i}=\left(\mathbf{n}^{i}\right)^{T}\left(\hat{\boldsymbol{\sigma}}+\sum_{j \neq i}^{N} \tilde{\boldsymbol{\sigma}}^{j}\right) \mathbf{b}^{i}
$$

where $\mathbf{n}^{i}$ is the normal vector to the glide plane of dislocation $i, \hat{\boldsymbol{\sigma}}$ is the stress field calculated from the boundary value problem, and $\tilde{\boldsymbol{\sigma}}^{i}$ is the stress field generated by dislocation $i$. Eq. (2) is used only when the Peach Koehler force is greater than the friction force. Otherwise, the dislocation is not moved at all. When a dislocation approaches the atomistic-DD interface (from the atomistic or the DD region), dislocations are passed across it via the insertion of a dislocation dipole. The insertion of a symmetric dislocation dipole that spans the interface and lies on the plane of the dislocation to be passed effectively moves the dislocation to the other side of the interface. The details of this procedure are given by Shilkrot et al. [15].

The CADD algorithm used here can be summarized as follows:

1. The forces on the atoms belonging to the interface and atomistic region are calculated and their positions are updated following a dynamic relaxation algorithm, with the pad atoms remaining fixed.

2. The position of the interface atoms is used to define the displacements of interface nodes which serve as Dirichlet boundary conditions for the continuum boundary value problem. The energy functional given in Eq. (1) is then minimized to obtain a solution using standard linear elastic finite element analysis.

3. If the Peach Koehler force acting on the dislocations are greater than the friction force, the position of the dislocations in the continuum region are updated following Eq. (2).

4. The positions of the pad atoms are updated in accordance with the continuum solution.

5. Return to step 1. 
A significant approximation in the current modeling framework is that the DD region can only accommodate in-plane displacement fields. This creates an inconsistency between the fully 3D atomistic and 2D DD regions. The effect of the inconsistency is that dislocations with a screw Burgers vector component relative to the planar FE region emitted from the crack tip experience a repulsive image force from the atomistic-DD interface. For the specific geometry and loading considered in this work, dislocations with screw character play a secondary role and their behavior has been shown to not be significantly affected by the artificial image forces by examining parametric studies involving the size and shape of the atomistic region. However, the incorporation of out-of-plane displacements in the DD region is important in general, and as such we plan to implement this feature in the future.

\subsection{Simulation setup}

Even though thermal activation is an important factor controlling fatigue crack growth in aluminum alloys [20], the simulations conducted in this study were performed in the zero temperature athermal limit. We do note that we have previously attempted to perform crack growth simulations in settings more consistent with ordinary experiments by using a combined time and spatial multiscale approach [21]. However, the methods employed to extend the timescale of the simulations were found to be too inefficient to reach ordinary experimental timescales when applied to the complex process of fatigue crack growth. In general, more reversibility is observed at the crack tip at colder temperatures, leading to higher threshold stress intensities [22].

The specimen consisted of an FCC crystal with an edge crack. The crystal lattice was oriented such that the horizontal axis corresponded with $[47 \overline{1}]$, the vertical axis with [113], and the out of plane direction with [21 1]. The crack was created by removing 3 consecutive planes of atoms. While the initial response of the crack is sensitive to the starting morphology, we expect the behavior beyond the first few events to be insensitive to the initial structure, as the crack tip configuration changes significantly as it is loaded. The crack plane was normal to the vertical axis with the crack front aligned with the [2 $\overline{1} 1$ ] direction. The ( $\overline{1} \overline{1} 1)$ slip plane intersected the crack plane at an angle of 58.5 degrees from the horizontal, with the [011] slip direction in the $x-y$ plane. The primary slip plane is the (1) 11$)$ plane and the primary slip direction is the [011] direction. All other slip planes are oblique to the $x-y$ plane, meaning the normal vector to the plane is not in the plane of the 2D DD region.

This orientation was chosen for three reasons. First, the primary means of slip is due to the nucleation and glide of full dislocations, even in the absence of thermal activation, making it consistent with general Al crack tip behavior at room temperature and experimental loading rates $[23,24]$. Second, the line direction of the full dislocations is normal to the crack front, meaning that there are no model thickness effects for the principal physical mechanisms. Third, the primary means of slip involves full dislocations that have pure edge character, consistent with the limitations of the CADD model used here.

Using CADD, the crack tip at the center of the specimen was embedded into a 3D atomistic region of approximately $150 \times 150 \times 10 \AA$, which was encompassed by a larger 2D plane strain, DD continuum region spanning $2 \times 2 \times 0.001 \mu \mathrm{m}$. The atomistic region uses the aluminum potential from Ercolessi and Adams [18], while the elastic constants in the continuum region match the the rotated elastic constants of the atomistic potential, $C_{11}=0.682, C_{12}=0.331, C_{44}=0.180 \mathrm{eV} / \AA^{3}$. The $0 \mathrm{~K}$ lattice constant of $4.03208 \AA$ was used. Loads were applied by prescribed displacements at the outer boundary of the DD region corresponding to the continuum solution for a crack in an anisotropic linear elastic material subjected to mode I loading. The affect of the far field $K_{I}$ loading on the changing crack tip position is assumed to be small, and less influential on crack tip evolution than the presence of the closest dislocations. The interface updating in CADD was done in every atomistic step. The simulation setup was the same for all simulations, with a range of different friction forces used for each loading condition. The simulations include prescribed loads of $\Delta K_{I}=0.4,0.5,0.6 \mathrm{eV} / \AA^{2.5}$ with $R=0$, and $\Delta K_{I}=0.2,0.3,0.4,0.5 \mathrm{eV} / \AA^{2.5}$ with $R=0.25$. Please refer to the Nomenclature for descriptions of the fatigue parameters.

The effect of alloying was modeled by the dislocation glide resistance in the DD model. As a simplification, a spatially homogeneous and constant glide resistance was used throughout the course of each simulation. In an actual alloy, the spatial heterogeneity of glide resistance, its evolution with plastic strain, and the direct interaction of alloying elements with the crack tip, may all play a role in the fatigue crack growth process. We characterize the simulations having 75.76, 94.33, 110, and $200 \mathrm{MPa}$ glide resistance as having a low friction force. 300, 400,500, and $600 \mathrm{MPa}$ as having a medium friction force, and $700,800,900$, and $1000 \mathrm{MPa}$ as having a high friction force. As a reference, the critical resolved shear stress of aluminum alloy 2024-T3 is around $115 \mathrm{MPa}$ [25], and the critical resolved shear stress of underaged Al-4\% Cu with primarily GP zone precipitates is around $76 \mathrm{MPa}$ [26], using standard arguments consistent with microscale simulation [27]. While the simulations with high glide resistances are not consistent with the bulk strengths of any Al alloys, they are applicable to situations where a fatigue crack is in a region of the material with a very high glide resistance, such as near a large intermetallic particle or grain boundary.

A single simulation with multiple loading cycles for each combination of load ratio, loading range, and friction force was performed. However, measurements of the crack growth and observed crack growth mechanisms in samples classified as high, medium, or low friction force were compiled from simulations of all loading ranges and load ratios for a given friction force classification. 


\section{Results and discussion}

The atomic mechanisms associated with fatigue crack extension as well as their dependence on dislocation glide resistance are documented here. As the friction force is increased, dislocations tend to remain closer to the crack tip, which changes the mechanisms of fatigue crack growth. To illustrate this point, Fig. 2 shows the positions of the discrete dislocation pileup for different friction forces after the completion of two cycles. Irreversible crack growth is observed for all friction forces, and is quantified as a function of the cyclic applied load and load ratio. The mechanisms associated with crack extension are presented below for low, medium, and high friction forces. After that, the crack growth data is presented to show the influence of the friction force on the near-threshold stress intensity range.

\subsection{Low friction force: increasing load}

At low friction forces, crack opening is often accompanied by edge dislocation nucleation along the (1 $\overline{1} 1)$ slip plane. When the opening portion of the loading cycle is started, multiple dislocations are nucleated on the primary slip plane. The nucleated dislocations break the bonds at the crack tip, causing the crack tip to change shape to a sharper

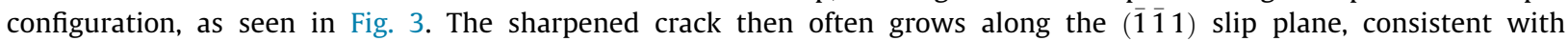
observations of Stage I fatigue crack growth in aluminum alloys. Driven by the applied load, the nucleated dislocations glide far away from the crack tip and thus do not appreciably change the effective stress intensity at the crack tip. Accordingly the transition from dislocation emission to brittle cleavage is likely a direct result of the crack tip shape change. This is consistent with the literature suggesting the importance of crack tip shape on crack tip deformation mechanisms [28-31].

Most commonly, cleavage crack growth is arrested when the crack tip reaches lattice defects created from previous cycles, as seen in Fig. 4. Such defects usually correspond to sessile dislocation cores or rows of missing atoms created by bond shifting at the crack surface. Most vacancy-like defects occur near the crack surface and are formed from the shifting of bonds in a coordinated manner. The vacancy-like defects observed far from the crack tend to be sessile dislocation cores.

The crack can also be stopped by emitting dislocations on the (111) plane, which is oblique to both the $x-y$ plane of the simulation and the original crack plane. These dislocations have low mobility due to the thin periodic structure of the simulation cell in the $[2 \overline{1} 1]$ direction. This geometry artificially forces these dislocations to form jogs so that they are periodic in the $[2 \overline{1} 1]$ direction. Dislocation emission on other oblique (111) planes was rarely observed. Due to their low mobility, dislocations emitted on oblique slip planes, whether at a propagating cleavage crack or a blunted crack tip, do not travel far from the crack tip. Thus they do not propagate to the atomistic boundary and are unaffected by CADD's inability to pass them into the DD region. They often interact with dislocations on the primary slip plane to create dislocation junctions and other lattice defects. In some cases, dislocations are nucleated on the primary slip plane from the oblique dislocation, a process which leaves behind a sessile Lomer dislocation at the nucleation site. We note that this mechanism has been observed using the same interatomic potential in a similar study at finite temperature and thicker sample sizes [21]. Furthermore, the nucleation of oblique dislocations at the crack tip tends to break bonds at the crack tip, and thus can be thought of as an additional mechanism for extending the crack in the (113) plane by a lattice spacing.

Alternatively, crack growth has also been observed to occur by the breaking of bonds not associated with cleavage or dislocation nucleation, but the shifting of bonds along the crack surface. This mechanism occurs at high loads, and is most likely an artifact of the thin specimen size and low temperature. Irregular atomic structures are present in Fig. 5 that have resulted from atoms shifting near the crack surfaces.

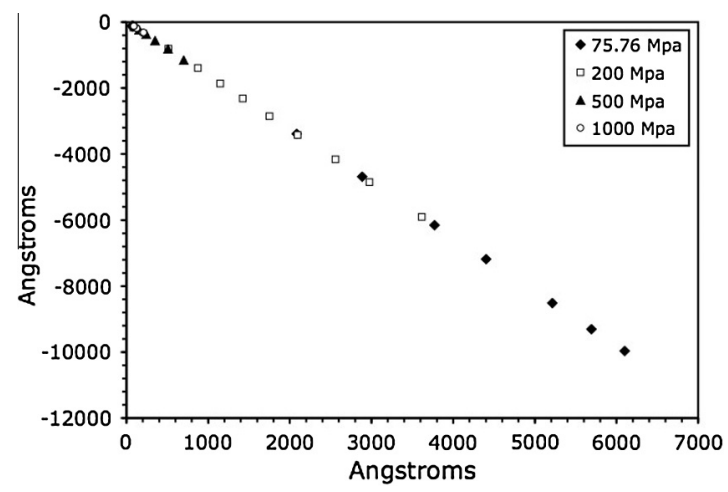

Fig. 2. Image showing the discrete dislocation positions in the bottom right quadrant of the CADD model, where dislocations glide into the DD region. The crack tip is at the origin of the image. The dislocation positions correspond to those at the end of the second cycle for various friction forces. The atomistic region extends to approximately $100 \AA$ on the horizontal axis and - $100 \AA$ on the vertical axis, and is thus not visible in the image due to its scale. 


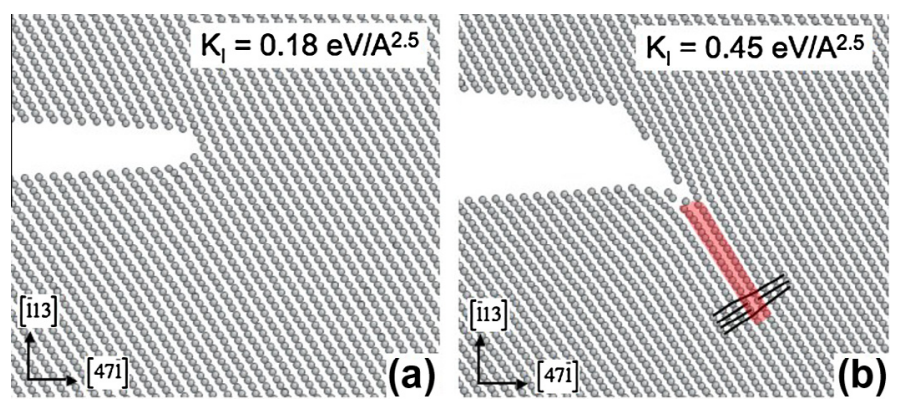

Fig. 3. Snapshot of simulation with $\Delta K_{I}=0.6 \mathrm{eV} / \AA^{2.5}, R=0$, and friction force $=110$ MPa showing crack tip sharpening by multiple dislocation emission in the first cycle. (a) The initial crack tip before any dislocations have nucleated. (b) The same crack tip after the nucleation of two full dislocations and a stacking fault. The highlighted area is a stacking fault created by the nucleation of a leading partial dislocation from the sharpened crack tip. The solid lines on the image show the slip created by the dislocation. The creation of a large stacking fault is rare since trailing partial dislocations are commonly nucleated in this orientation. All images of the atomistic region are plotted with AtomEye [38].

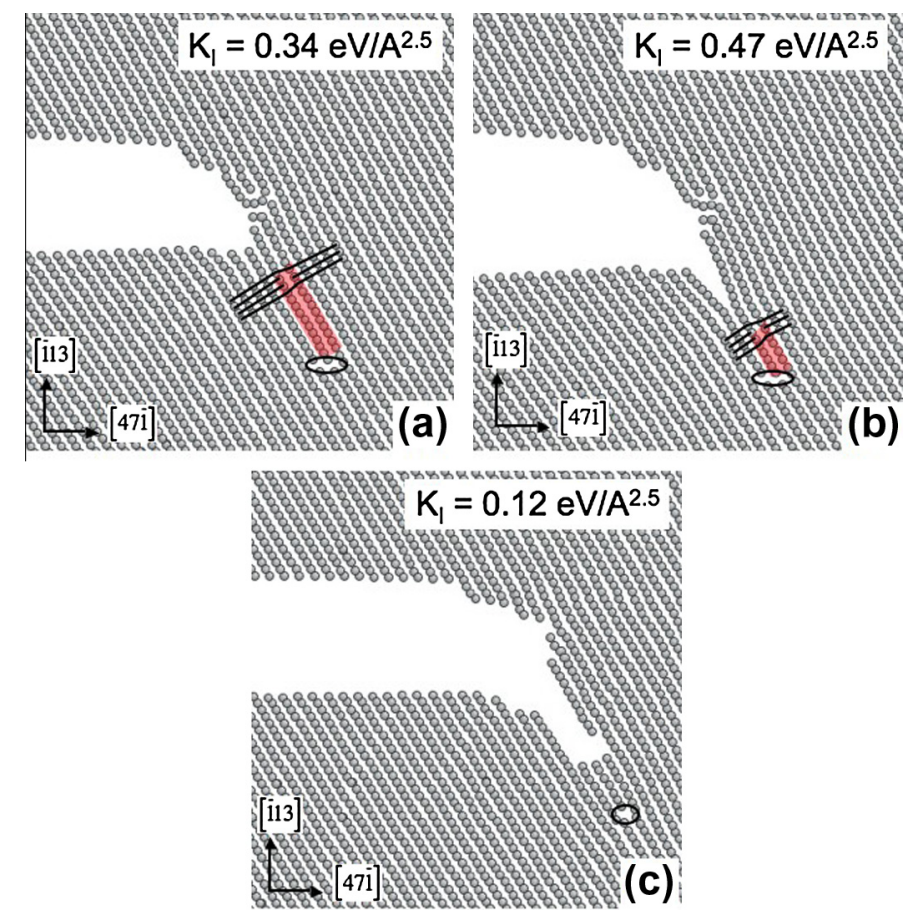

Fig. 4. Snapshot of simulation with $\Delta K_{I}=0.6 \mathrm{eV} / \AA^{2.5}, R=0$, and friction force $=700 \mathrm{MPa}$ showing effects of lattice defects near the crack tip in the second cycle. (a) The crack tip has nucleated a full dislocation that propagates away from the tip, but is stopped by a defect. The defect, circled by a solid line, contains some increment of slip on an oblique slip plane. The highlighted area is a stacking fault created by the nucleation of a leading partial dislocation. The solid lines on the image show the slip created by the dislocation. The slip near the defect is difficult to visualize, thus is not shown with guiding lines. (b) After the dislocation has stopped, the crack tip begins to propagate along the (1 11 ) plane. The defect also arrests the crack, which blunts by nucleating dislocations on oblique slip planes instead of propagating further. (c) After reaching the peak load of the second cycle, the system is unloaded. The snapshot shows the extended and blunted crack tip, and the lattice defect circled with a solid line.

\subsection{Low friction force: decreasing load}

When unloading the crack from the deformed configuration at low friction forces, several mechanisms were observed to lead to irreversible crack growth during a loading cycle. When a propagating crack on the inclined slip plane nucleates dislocations on the oblique slip planes, many of the dislocations do not return to the crack, and the crack remains open after the load is removed. The oblique dislocations do not return because, as mentioned earlier, the dislocations cannot be projected to a point in the $x-y$ plane. However, since the atomistic region is thin, they create a jog to become periodic in the $z$ direction. This jog greatly reduces their mobility when gliding away from or towards the crack tip. Furthermore, since oblique dislocations seldom return to the crack, dislocations that are emitted from the primary slip plane may return to a different configuration than they left causing permanent deformation. Also, if a crack propagates into a defect, when the load is removed 

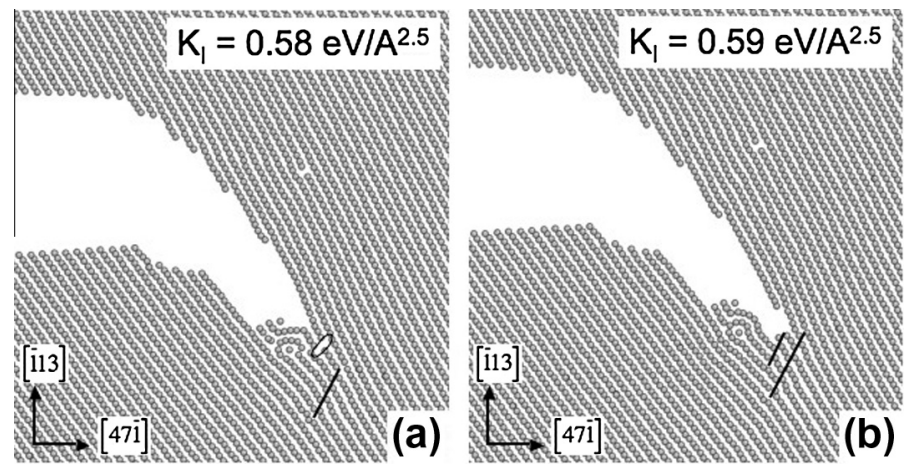

Fig. 5. Snapshot of simulation with $\Delta K_{I}=0.6 \mathrm{eV} / \AA^{2.5}, R=0$, and friction force $=400 \mathrm{MPa}$ showing the crack opening along a lattice defect in the second cycle. Bond breaking and shifting of the lattice at the crack surface is observable on the lower crack face near the crack tip. (a) The crack has a sharp tip, and a small void is present near the tip, circled with a solid line. Solid straight lines indicate slip on oblique slip planes. (b) The crack has opened along the slip plane and connected with the lattice defect. Again, straight lines indicate slip on planes oblique to the crack plane.

the crack will not return to its original configuration, as seen in Fig. 4. At high loads, the bond shifting may not be fully reversible if the crack nucleates a dislocation or the crack grows after the atoms have shifted. In a few cases the crack tip returned to its original shape, leaving no permanent deformation. This occurred when a crack on the inclined slip plane did not nucleate blunting dislocations, or when the crack tip only produced dislocations that returned to the crack after the load was removed.

\subsection{Medium friction force: increasing and decreasing load}

At medium friction forces, the crack opening mechanisms are for the most part identical to the low friction force simulations. The major difference is that dislocations nucleated on the primary slip plane eventually shield the tip from further nucleation. Fig. 6 shows two identical simulations with different friction forces, where one crack tip is shielded and another is not. If shielding occurs, dislocations on oblique slip systems are nucleated, leading to bond breaking in the original crack plane, and the creation of numerous defects around the crack tip. The oblique dislocations can produce a high number of local defects because they rarely return to crack. However, they remain close to the tip and can combine with dislocations on the primary slip plane to form Lomer locks. In some cases an oblique edge dislocation can nucleate a Lomer lock and full dislocation on primary slip plane as observed by Baker and Warner [21]. Since dislocations are still emitted along the primary slip plane, some crack sharpening and cleavage along the slip plane are observed. However, the subsequent crack growth is influenced by nearby defects. Fig. 5 shows an instance where a nearby defect promoted crack growth while Fig. 4 is an example where a nearby defect arrested crack growth. Depending on the core structure and burgers vector of the defect, as well as the position of the crack tip with respect to the defect, crack growth can either be enhanced or inhibited. For example, a crack approaching the compressive lobe of the strain field of a Lomer dislocation might be arrested, while

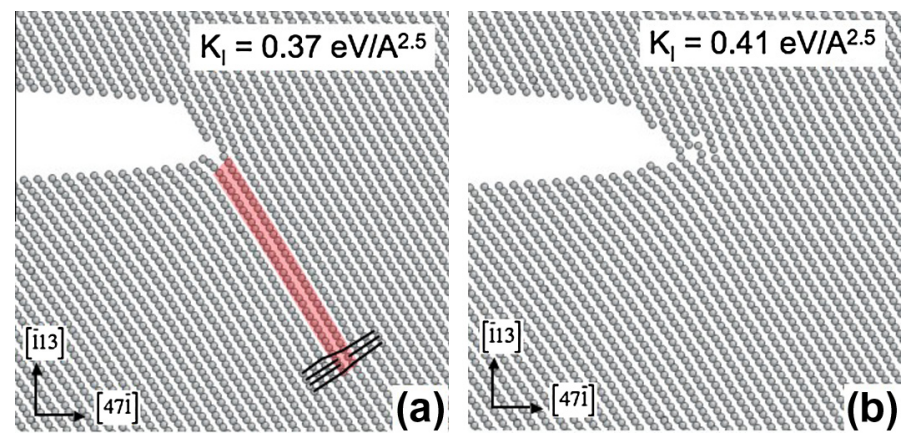

Fig. 6. Snapshot of simulation with $\Delta K_{I}=0.6 \mathrm{eV} / \AA^{2.5}$ and $R=0$ showing crack tip shielding by the initial nucleated dislocation in the first cycle. The subsequent crack tip behavior is controlled by friction force, i.e. the distance of the initial dislocation from the crack tip. (a) Simulation with friction force $=110 \mathrm{MPa}$. The initial dislocation has travelled over $1300 \AA$ from the crack tip along the slip plane, allowing the crack to nucleate a second dislocation in the same slip system. Notice that the crack grows by a small increment along the (113) plane by bonds breaking from the nucleation of the dislocation. The highlighted area is a stacking fault created by the nucleation of a leading partial dislocation from the sharpened crack tip. Like Fig. 3 , the creation of a large stacking fault is rare in comparison to the nucleation of full dislocations at higher loads. The solid lines on image show the slip created by the dislocation. (b) Simulation with friction force $=500 \mathrm{MPa}$. The initial dislocation remains close to the crack tip (within $500 \AA$ along the slip plane). Instead of nucleating a dislocation, bonds break at the crack tip extending the crack by a few Angstroms along the original crack plane. 
a crack approaching the tensile lobe may be accelerated. Bond shifting still occurs at high loads, especially when the crack has several defects in an area of high strains, as in Fig. 5. Upon unloading, the mechanisms causing irreversible crack growth are the same, but the influence of defects are more pronounced since they are more abundant when the friction force is raised. Again, reversible closing of the crack is observed if dislocations remaining near the crack are absorbed back into the crack surfaces.

\subsection{High friction force: increasing and decreasing load}

At high friction forces, dislocations do not propagate far from the crack, and new dislocations are not nucleated on the primary slip plane due to dislocation shielding. The nucleation of oblique dislocations is very common and breaking bonds move the crack forward along the original crack plane, reminiscent of an atomic scale Stage II fatigue crack growth. Defects and nucleated dislocations remain very close to the crack tip. The amount of crack growth is usually smaller at the highest friction forces since the deformation does move away from the crack and usually returns to the crack after the load is removed. When unloaded, irreversible deformation is observed only if dislocations or defects are not absorbed back into the crack.

As mentioned previously, we caution that the mechanisms observed in these simulations must be interpreted with care when comparing to ordinary materials due to the athermal and near-2D nature of the simulations. The near-2D nature of the simulations can affect the results in a multitude of ways, two of which we deem worth noting here. First, a single defect near the crack tip in the simulations will behave as a dense row of defects due to the periodic boundary conditions. Thus, crack tip interactions with nearby defects are likely magnified relative a fully 3D specimen. Second, dislocations that have a line orientation that is not parallel with the out of plane direction will have a high density of jogs, making dislocation mobility much lower than it would be in a fully 3D case.

The lack of thermal activation, in the zero temperature simulations, requires higher loads to nucleate dislocation from the crack tip which would in general increase the stress intensity required for crack growth relative to ordinary experimental conditions. This conclusion is consistent with experimental observations [22]. It is worth noting that the complex dislocation reactions observed here, like dislocations changing slip planes to propagate in directions of higher resolved shear stress, are consistent with observations from thicker specimens at finite temperature [21].
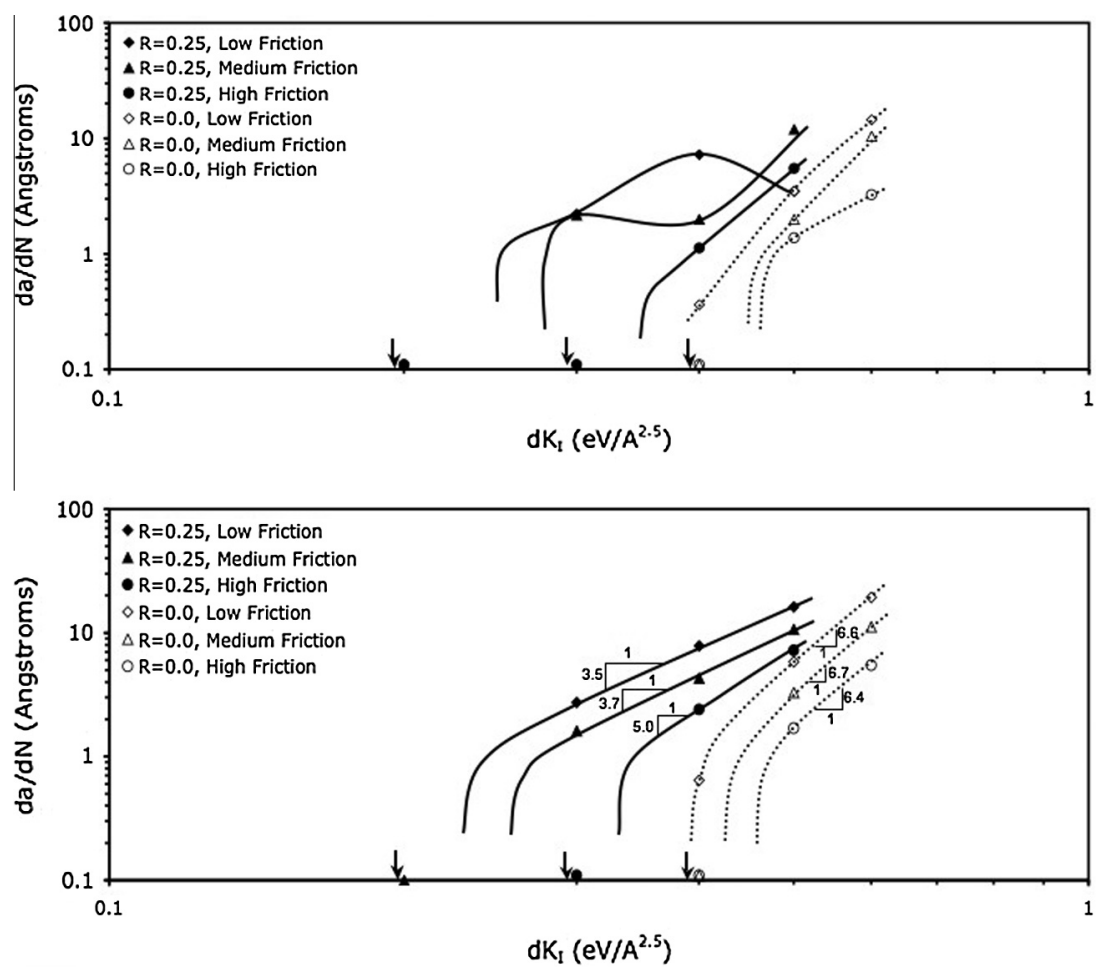

Fig. 7. Plot of the crack growth per cycle, da/dN, as a function of the stress intensity range, $\Delta K_{I}$, load ratio, $R$, and the friction force. The data corresponds to the average crack growth over as many cycles as were simulated at the given stress intensity range, friction force, and load ratio. The bottom plot includes crack growth values from all recorded cycles, while the top plot excludes data from the first cycle. The lines through the data are drawn to guide the eye and are not analytical functions. The downward arrows near data points indicate below-threshold values. The data suggests that the threshold stress intensity range decreases as the friction force decreases. 


\subsection{Crack growth per cycle}

The fatigue crack growth averaged over all recorded cycles, and all but the first cycle, is compiled for each $\Delta K_{I}$ and $R$ in Fig. 7. A single simulation with multiple cycles was performed at each combination of load ratio, loading range, and friction force. However, the average crack growth per cycle is compiled from all simulations classified as high, medium, or low friction force. Furthermore, the crack growth is measured along the crack path, not along the horizontal direction as in largerscale experiments. The position of the crack tip was determined by inspection. It was defined using the atom on the crack surface farthest from a fixed reference point on the original crack plane or inclined slip plane. Since the first cycle usually produces an anomalously large crack growth increment, the averaged data for all but the first cycle is a more representative value for the average fatigue crack growth per cycle. The average value including the first cycle provides more data and smoother curves, but it also increases the amount of crack growth recorded. The total number of cycles simulated ranged from 3 to 10 depending on the total crack growth accumulated over all of the cycles. Once the crack approached the atomistic-DD interface, the simulations were stopped. Only three cycles were recorded for $\Delta K_{I}=0.6 \mathrm{eV} / \AA^{2.5}$ with $R=0$ and $\Delta K_{I}=0.5 \mathrm{eV} / \AA^{2.5}$ with $R=0.25$, around 5 cycles were used for $\Delta K_{I}=0.4-0.5 \mathrm{eV} / \AA^{2.5}$ with $R=0$ and $\Delta K_{I}=0.4 \mathrm{eV} / \AA^{2.5}$ with $R=0.25$, and around 10 cycles were simulated for $\Delta K_{I}=0.2-0.3 \mathrm{eV} / \AA^{2.5}$ with $R=0.25$. The most variability occurs in results averaged over the fewest cycles. Since four individual friction force values were grouped together for low, medium, and high friction data points, at least 12 (or 8 when not including the first cycle) cycles are included in each data point.

The data shows an expected shift in $\Delta K_{I}^{\text {th }}$ with an increase in $R$, and an increasing amount of crack growth per cycle as the stress intensity is raised above $\Delta K_{I}^{\text {th }}$. The data also indicates that the presence of a higher friction force raises $\Delta K_{I}^{\text {th }}$, similar to increasing $R$. The trend is clearer when examining the data that includes the first cycle, however, it holds regardless of whether the first cycle is included. To compare with experimental measurements and other modeling predictions in the literature, the data can be described with a power law function, $\log d a / d N$ versus $\log \Delta K_{I}$. The best-fit slope of the curves (the power law exponent) ranges between 6.4-6.7 for $R=0$ and 3.5-5.0 for $R=0.25$. For comparison, DD simulations predict power law exponents of 6.33 for $R=0.1,6.46$ for $R=0.3$ and 8.55 for $R=0.5$ [10]. The DD simulations in the literature differ from the simulations performed here in that dislocations are emitted from sources in the former and from the crack tip in the latter. Additionally, the DD simulations require a crack growth law as input. Fatigue experiments on metals and metallic alloys range greatly but are around 3 [32] or 4 [33] for metals and 2 for many alloys [33].

In general, the simulations predict that an increasing resistance to dislocation glide increases the reversibility of the cyclic crack growth process, and thus reduces the fatigue crack growth rate. This result contradicts experimental data that shows a decrease in the fatigue crack growth threshold with artificial aging treatment, when the hardness of the alloy is increased [33]. We hypothesize that this contradiction may result from the absence of two key features in our model. The first is that increased microcracking may occur with increased aging due to the formation of large precipitates. The microcracking may facilitate enhanced fatigue crack growth, counteracting of dislocation glide resistance observed in the model. Second, the spatial heterogeneity of dislocation glide resistance will increase with aging as alloying atoms come out of solution to form precipitates and precipitates grow by Oswald ripening. While the average dislocation glide resistance can increase with aging, the glide resistance in local regions of material will decrease as precipitate free regions around each precipitate grow. Thus, it is possible that the enhanced fatigue crack growth in these regions, due to the increased mobility of dislocations, dominates the overall change in threshold fatigue crack growth rates. The idea of a decreasing fatigue threshold with increasing heterogeneity was also put forth by Suresh et al. [34] to describe experimentally observed crack growth rates in aluminum alloy 7075. In that work, the threshold stress intensity range decreased as the spacing between precipitates increased as the alloy was aged. Consistent with this idea is the experimental work of Laird and Thomas [35] who have also observed larger crack growth rates when the size of precipitate-free regions of $\mathrm{Al}-4 \% \mathrm{Cu}$ are increased by aging.

\section{Summary and conclusions}

Using a concurrent multiscale simulation approach combining atomistic simulation and discrete dislocation dynamics, the atomic scale mechanisms associated with fatigue crack growth have been investigated and the effect of dislocation glide resistance has been studied. The simulations suggest that dislocation emission along the primary slip plane is the primary mechanism that occurs during a fatigue load cycle. The dislocation emission can change the crack tip geometry and the near tip stress field (from nearby dislocations) and ultimately lead to crack growth. Crack growth was observed to occur either on a low energy crystallographic plane by cleavage, or on the crack plane by individual bond breaking events at the crack surface, as can result from dislocation nucleation. The irreversible character of fatigue crack growth resulted from the interaction of the crack with lattice defects and the emission of dislocations from the crack tip that did not return upon unloading.

The predominant fatigue crack growth mechanisms varied as a function of the prescribed dislocation glide resistance. When the glide resistance is small, the simulations suggest that sharpening of the crack tip occurs by multiple dislocation emission on the primary slip plane. The sharpening of the tip eventually induces crack propagation on the primary slip plane, an observation consistent with the sudden crack growth and shear-band formation observed in copper [4]. Our model predicts that a propagating sharp crack will arrest when it reaches a region of material where dislocation emission can resume, blunting the tip. The above two steps can then repeat. The crack tip remains open upon unloading, leading to permanent crack growth, when the blunting dislocations glide far enough away from the crack tip that they do not return during 
unloading. When no blunting occurs, the crack usually closes in a reversible manner. The model predictions of crack growth along crystallographic planes (including the dominant planes) is consistent with previous atomistic modeling of magnisium [7] and copper [6].

When a medium glide resistance is applied in the model, emitted dislocations remained closer to the crack tip, providing more shielding. This led to the nucleation of dislocations on oblique slip planes or bond breakage at the crack tip. This extends the crack tip and introduces defects close to the tip, which can block or aid crack growth. Since some defects and dislocations remain close to the tip, some deformation is reversible when the applied load is reduced. Crack tip sharpening was also observed, but is less common. The creation of lattice defects, like vacancies and voids, is very common in the literature [2-8]. However, no large voids were created in this work as is observed by others [2-8].

At the highest glide resistances, the model suggests that emitted dislocations provide significant shielding of the crack tip. This leads to crack extension primarily by bond breaking and oblique dislocation emission. It also introduces a large number of defects that remain close to the crack tip. Since dislocations and defects do not travel far from the crack, much of the deformation is reversible. In all simulations, plasticity is confined to one or two slip systems, creating persistent slip bands, consistent with the literature [2-8].

For all dislocation glide resistances examined, the model predicts that oblique dislocations and lattice defects remain near the crack tip. These dislocations and defects often interact with dislocations emitted on the primary slip plane and leave permanent junctions or lattice defects. In many cases, the model suggests that lattice defects act to promote the extension of the crack, and when unloaded return to a different configuration, creating permanent crack growth.

The changing crack growth mechanisms indicate that an increasing dislocation glide resistance leads to a transition from crystallographic crack growth with plasticity occurring on a primary slip system to crack growth on the original crack plane with plasticity occurring on multiple slip systems. Increasing the dislocation glide resistance increases the proximity of the nucleated dislocations to the crack tip, and thus, causes the threshold stress intensity to increase. With this result being contrary to experimental observations, we suggest that it indicates the importance of features not included in the model, such as microcracking at large precipitates [36] and the spatial heterogeneity of the dislocation glide resistance [37,34]; thus, providing sound motivation for future work. Despite these short comings of the present model, we assert that this work represents a step towards the development of atomistic based fatigue crack growth laws in real materials, which could be used in mesoscale modeling for the generation of physics based crack growth laws in macroscopic simulation.

\section{Acknowledgements}

The authors gratefully acknowledge support from Ed Glaessgen and Steve Smith at NASA (Grant No. NNX08BA39A), Paul Hess at the Office of Naval Research (Grant No. N000141010323), and Ali Sayir at the Air Force Office of Scientific Research (Grant No. FA95501110273).

\section{References}

[1] Farkas D, Duranduru M, Curtin W, Ribbens C. Multiple-dislocation emission from the crack tip in the ductile fracture of Al. Philos Mag A 2001;81:1241-55.

[2] Nishimura K, Miyazaki N. Molecular dynamics simulation of crack growth under cyclic loading. Comput Mater Sci 2004;31:269-78.

[3] Farkas D, Willemann M, Hyde B. Atomistic mechanisms of fatigue in nanocrystalline metals. Phys Rev Lett 2005;94. 165502-1-4.

[4] Potirniche GP, Horstemeyer MF, Jelinek B, Wagner GJ. Fatigue damage in nickel and copper single crystals at nanoscale. Int J Fatigue 2005;27:1179-85.

[5] Potirniche GP, Horstemeyer MF. On the growth of nanoscale fatigue cracks. Philos Mag Lett 2006;86:185-93.

[6] Potirniche GP, Horstemeyer MF, Gullett PM, Jelinek B. Atomistic modelling of fatigue crack growth and dislocation structuring in FCC crystals. Proc Roy Soc A 2006;462:3707-31.

[7] Tang T, Kim S, Horstemeyer MF. Fatigue crack growth in magnesium single crystals under cyclic loading: molecular dynamics simulation. Comput Mater Sci 2010;48:426-39.

[8] Horstemeyer MF, Farkas D, Kim S, Tang T, Potirniche G. Nanostructurally small cracks (NSC): a review on atomistic modeling of fatigue. Int J Fatigue 2010;32:1473-502.

[9] Deshpande V, Needleman A, Giessen EVD. A discrete dislocation analysis of near-threshold fatigue crack growth. Acta Mater 2001;49:3189-203.

[10] Deshpande V, Needleman A, Giessen EVD. Discrete dislocation modeling of fatigue crack propagation. Acta Mater 2002;50:831-46.

[11] Deshpande V, Needleman A, Giessen EVD. Discrete dislocation plasticity modeling of short cracks in single crystals. Acta Mater 2003;51:1-15.

[12] Deshpande V, Needleman A, Giessen EVD. Scaling of discrete dislocation predictions for near-threshold fatigue crack growth. Acta Mater 2003;51:4637-51.

[13] Chen Q Deshpande V, Giessen EVD, Needleman A. Friction stress effects on mode I crack growth predictions. Scr Mater 2003;48:755-9.

[14] Shilkrot LE, Miller RE, Curtin WA. Coupled atomistic and discrete dislocation plasticity. Phys Rev Lett 2002;89:1-4.

[15] Shilkrot LE, Miller RE, Curtin WA. Multiscale plasticity modeling: coupled atomistics and discrete dislocation mechanics. J Mech Phys Solids 2004;52:755-87.

[16] Nair AK, Warner DH, Hennig RG, Curtin WA. Coupling quantum and continuum scales to predict crack tip dislocation nucleation. Scr Mater 2010;63:1212-5.

[17] Nair AK, Warner DH, Hennig RG. Coupled quantum-continuum analysis of crack tip processes in aluminum. J Mech Phys Solids 2011;59:2476-87.

[18] Ercolessi F, Adams JB. Interatomic potentials from 1st-principles calculations - the force-matching method. Europhys Lett 1994;26(8):583-8.

[19] van der Giessen E, Needleman A. Discrete dislocation plasticity: a simple planar model. Model Simul Mater Sci Engng 1995;3:689-735.

[20] Wei R. Fatigue-crack propagation in a high-strength aluminum alloy. Int J Fract Mech 1968;4:159-68.

[21] Baker K, Warner D. Extended timescale atomistic modeling of crack tip behavior in aluminum. Model Simul Mater Sci Engng 2012;20:065005.

[22] McKittrick J, Liaw P, Kwun S, Fine M. Threshold for fatigue macrocrack propagation in some aluminum alloys. Metall Trans A 1981;12A:535-1539.

[23] Warner D, Curtin W, Ou S. Rate dependence of crack-tip processes predicts twinning trends in FCC metals. Nat Mater 2007;6:876-81.

[24] Warner DH, Curtin WA. Origins and implications of temperature-dependent activation energy barriers for dislocation nucleation in face-centered cubic metals. Acta Mater 2009;57:4267-77. 
[25] Data MMP. Aluminum 2024-t3. <www.matweb.com/search/SpecificMaterial.asp?bassnum=MA2024T3> [Accessed 06.02.13]. Provided by the Aluminum Association, Inc.

[26] Silcock J, Heal T, Hardy H. Structural ageing characteristics of binary Al-Cu alloys. J Inst Met 1953-1954;82:239-48.

[27] Singh CV, Warner DH. An atomistic-based hierarchical multiscale examination of age hardening in an Al-Cu alloy. Metall Mater Trans A 2013;44:2625-44.

[28] Beltz GE, Lipkin DM, Fischer LL. Role of crack blunting in ductile versus brittle response of crystalline materials. Phys Rev Lett 1999;82:4468-71.

[29] Fischer LL, Beltz GE. The effect of crack blunting on the competition between dislocation nucleation and cleavage. J Mech Phys Solids 2001;49:635-54.

[30] Tadmor E, Hai S. A Peierls criterion for the onset of deformation twinning at a crack tip. J Mech Phys Solids 2003;51:765-93.

[31] Hai S, Tadmor E. Deformation twinning at aluminum crack tips. Acta Mater 2003;51:117-31.

[32] Frost N, Pook L, Denton K. A fracture mechanics analysis of fatigue crack growth data for various materials. Engng Fract Mech 1971;3:109-26.

[33] Suresh S. Fatigue of materials. Cambridge University Press; 1998.

[34] Suresh S, Vasudevan A, Bretz P. Mechanisms of slow fatigue crack growth in high strength aluminum alloys: role of microstructure and environment. Metall Trans A 1984;15A:369-79.

[35] Laird C, Thomas G. On fatigue-induced reversion and overaging in dispersion strengthened alloy systems. Int J Fract 1967;3:81-97.

[36] Sharma V, Kumar KS, Rao BN, Pathak S. Fatigue crack growth of AA2219 under different aging conditions. Mater Sci Engng: A 2011;528:4040-9.

[37] Wanhill R. Low stress intensity fatigue crack growth in 2024-T3 and T351. Engng Fract Mech 1988;30:233-60.

[38] Li J. Atomeye: an efficient atomistic configuration viewer. Model Simul Mater Sci Engng 2003;11:173-7. 